\title{
A Method of Firing Nearly Invisible 2D Code with Public and Secret Information on Ceramic Products
}

\author{
Masaki Fujikawa a, ${ }^{\mathrm{a},}$, Yui Ueno ${ }^{\mathrm{a}}$, Daiji Osawa ${ }^{\mathrm{b}}$, Eigo Nishimura ${ }^{\mathrm{c}}$, Koutaro Gomi ${ }^{\mathrm{d}}$, \\ Hideki Iwasaki ${ }^{\mathrm{d}}$, Toshiaki Harada ${ }^{\mathrm{e}}$, and Naoki Adachi ${ }^{\mathrm{e}}$ \\ ${ }^{a}$ Kogakuin University, Nishi-Shinjyuku 1-24-2, Tokyo 163-8677, Japan \\ ${ }^{\text {b}}$ Takane Silk Co., Ltd., Takane-cho 4-6, Tajimi, Gifu Pref. 507-0078, Japan \\ 'Science Park Corporation, Iriya 3-1649-2, Zama, Kanagawa Pref. 252-0024, Japan \\ 'Yamaka Shoten Ltd., Izumi-cho Kujiri 547, Toki, Gifu Pref. 509-5142, Japan \\ ${ }^{\text {e}}$ Gifu Prefectural Ceramics Research Institute, Hoshigadai 3-11, Tajimi, Gifu Pref. 507-0811, Japan \\ *Corresponding Author: clavecin@yahoo.co.jp
}

\begin{abstract}
In this paper, we introduce a method for firing nearly invisible, two-dimensional (2D) code on ceramic product surfaces and a 2D code-reader system, equipped with a smartphone and an ultraviolet (UV) light, to extract encoded character information (public and encrypted secret information). We created the nearly invisible 2D code to add character information to ceramic surfaces without impairing the ceramics' colors and patterns as much as possible. When irradiated by UV light, the code is visible to the naked eye, emitting a pale-blue light. The 2D code-reader system uses a smartphone camera and a 2D code-recognition algorithm to extract character information from the 2D code reflected on the smartphone's display. However, only smartphones that have access to digital keys can decrypt the encrypted secret information. We successfully fired this 2D code on ceramic surfaces.
\end{abstract}

Keywords: Secure Quick Response Codes, public information, secret information, nearly invisible 2D code.

\section{Introduction}

\subsection{Background and target}

Today, adding 2D codes onto the surfaces of everyday products, such as home appliances, foods, and tools, is not uncommon because of the proliferation of codes. Consumers can access product information publicly released by a manufacturer-such as the product's name, the manufacturer's name, and quality management information - by using a smartphone with a 2D code-reader application (app). With this capability, consumers can deepen their sense of trust in products and manufacturers. Conversely, by disclosing product information, manufacturers can be transparent with their customers.

In general, 2D codes are added onto product surfaces so that customers easily recognize them. However, such codes are typically not added onto certain products - such as ceramics-because the patterns and colors would be negatively affected. However, the aforementioned, mutually beneficial relationship between manufacturers and consumers would be further strengthened if a nearly invisible 2D code could be added onto these products without hindering their patterns and colors. The codes would only be visible when consumers want to refer to or access product information.

A nearly invisible 2D code could be acceptable in the ceramics market. This belief was derived from interviews conducted with some merchants $(\mathrm{n}=109)$ who visited a trade fair held in Nagoya, Japan, in 2018. Of the 109 interviewees, 49 were in favor of a $2 \mathrm{D}$ code with the above-mentioned features. Considering the customers' viewpoints, they commented that, while an invisible 2D code would not affect a product's patterns and colors, the customers would be unable to quickly find the code when they wanted to refer to or access the product's information. However, if the 2D code was nearly invisible, it would not significantly impact the product's patterns and colors, and customers could find the code relatively easily. 


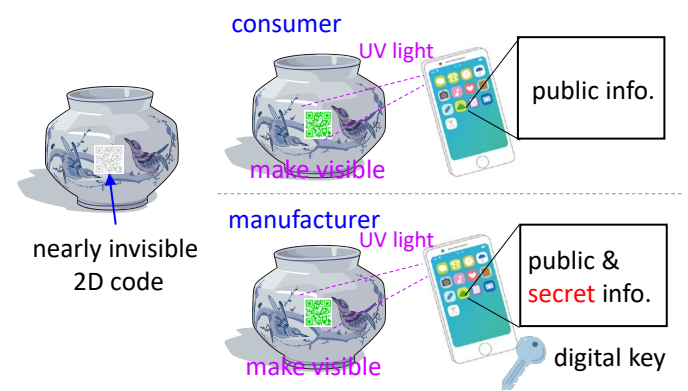

Fig. 1. An illustration of the 2D code and smartphone-based reader system. The nearly invisible 2D code becomes visible in response to the presence of UV light.

The merchants also provided further feedback to add a security feature to genuine ceramic products, as counterfeit products have been distributed in the market. The development of technical structures could enable manufacturers to authenticate products when they receive queries from consumers or merchants regarding the product's authenticity. The bothersome operation of matching the product to a manual describing the authenticity validation method should be omitted to save time.

In this paper, we focus on ceramics as one product whose patterns and colors are valued. We propose a method of baking nearly invisible, secure 2D code (called Secure Quick Response Codes, or SQRC ${ }^{(1)}$ ) that can store public and encrypted secret information in ceramic surfaces. We also propose a smartphone-based reader system that can make the fired $2 \mathrm{D}$ code clearly visible and extract character information (public and secret information) from the code (Fig. 1). We set the product information as public, and the authenticity verification method as secret, so that only manufacturers can access each product's secret information. Because products are linked to corresponding public and secret information via the $2 \mathrm{D}$ code, manufacturers can omit the above-mentioned matching operation when they are authenticating products.

This paper consists of four sections. In Section 2, we describe the method for baking nearly invisible SQRC onto ceramic surfaces. In the same section, we also describe the smartphone-based SQRC reader system and the process for making the nearly invisible code visible. Considerations are described in Section 3. We conclude this paper in Section 4.

\subsection{Preconditions}

(1) We set the size of the SQRC fired onto the ceramic surfaces as $1.5 \mathrm{~cm}^{2}$. This dimension is based on the ba-
Table 1. Job positions and corresponding authentication methods.

\begin{tabular}{|c|c|}
\hline Job position & Authentication method \\
\hline Staff & $/ \mathrm{n} 1 / \mathrm{sec} 1 /$ index.html \\
\hline \multirow{2}{*}{ Chief } & $/ \mathrm{n} 1 / \mathrm{sec} 1 /$ index.html \\
& $/ \mathrm{n} 1 / \mathrm{sec} 2 /$ index.html \\
\hline \multirow{3}{*}{ Section Chief } & $/ \mathrm{n} 1 / \mathrm{sec} 1 /$ index.html \\
& $/ \mathrm{n} 1 / \mathrm{sec} 2 /$ index.html \\
& $/ \mathrm{n} 1 / \mathrm{sec} 3 /$ index.html \\
\hline
\end{tabular}

sic size of the signature and seal (called "rakkan" in Japanese, a kind of trademark that can distinguish manufacturers) that is fired onto ceramic surfaces.

(2) We adopt the SQRC's error-correction level as level M (approx. 15\%) because it is generally used among printed standard materials with Quick Reaction (QR) codes and SQRCs ${ }^{(2)}$. We also adopt an alphanumeric character mode for the SQRC text, as this mode can more easily express Uniform Resource Locators (URLs) compared to other character modes. It allows verifiers to access, via smartphone, both public and secret information, while consumers can only access public information.

(3) The secret information stored in the SQRC is set to be longer than the public information. The secret information should not be available to the public because counterfeiters could refer to this information to make cleverly copied products. As shown in Table 1, we set multiple verifiers with different levels of authority (they refer to more information than do consumers to authenticate products). Staff (those who perform basic verification of a product's authenticity) can only refer to $/ n 1 / \mathrm{sec} 1 /$ index.html, while the section chief (individual who conducts detailed authentication) can refer to the entire index.html. As an example of a concrete verification method, $/ n 1 / \mathrm{sec} 1 /$ index.html could have the following directions: "Check the observation point A. If this point becomes illuminated while receiving UV light of wavelength $\mathrm{B}$, then the product appears to be genuine." Other index.html could have different observation points and wavelengths for the chief and section chief to check the product's authenticity.

(4) To render the nearly invisible SQRC invisible, consumers and verifiers have their own UV light. Although UV lights are available in the market at an affordable rate, consumers could access product information more easily if manufacturers packaged a UV light with the ceramic products. 
(5) Regarding reading and extracting character information from the $\mathrm{SQRC}$, consumers use a $\mathrm{QR}$ code-reader app, distributed by Denso Wave Incorporated, while verifiers use a SQRC reader app developed by the authors. Because both apps have the same QR code and SQRC recognition algorithm, read and extraction performance are the same.

\section{Forming 2D code on ceramics}

A printing technique (screen printing and transfer sheet) is used to fire patterns (SQRCs) onto the ceramics. In this section, we describe how the $2 \mathrm{D}$ code is created, printed, and fired.

\subsection{Creating the SQRC}

The SQRC, one of the 2D codes, was developed by Denso Wave Incorporated with a QR code. As shown in Fig. 2 , the SQRC's appearance is almost the same as that of the QR code. The SQRC can store both public and encrypted secret information, while the QR code can store only public information. Encryption operations can be executed in smartphones or cloud environments, but a digital key is needed in both cases. Hence, smartphones without the appropriate digital key cannot decrypt the encrypted secret information.

The amount of public and encrypted secret information stored in the SQRC depends on a combination of the error-correction level (level L: $\sim 7 \%$, level M: 15\%, level Q: $\sim 25 \%$, and level $\mathrm{H}: \sim 30 \%$ ), character mode (numerical, alphanumerical, binary, or kanji character mod e), and the version (a number of black and white colored

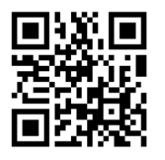

Fig. 2. An example of the SQRC (version 2). Public information with 22 characters and encrypted secret information with 6 characters are included.

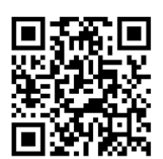

Fig. 3. Another SQRC example (Ver. 3). Public information, consisting of 22 characters, and encrypted secret information, consisting of 29 characters, are included.
Table 2. URLs and corresponding target individual(s).

\begin{tabular}{|l|l|}
\hline \multicolumn{1}{|c|}{ URLs } & \multicolumn{1}{|c|}{ Target Individual(s) } \\
\hline [public] www.abc123.co.jp/n1 & $\begin{array}{l}\text { Consumer and } \\
\text { Verifier (Staff, Chief, } \\
\text { \& Section Chief) }\end{array}$ \\
\hline$[$ secret] $/ \mathrm{sec} 1$ & Verifier \\
\hline$[$ secret $] /$ sec2 & $\begin{array}{l}\text { Chief and Section } \\
\text { Chief }\end{array}$ \\
\hline$[$ secret $] / \sec 3$ & Section Chief \\
\hline
\end{tabular}

cells that form the SQRC code, from Ver. 1 (21 times 21 cells) to Ver. 40 (177 times 177 cells). The larger the size of the cell (or the smaller the version number becomes), the more easily the SQRC can be read.

For an SQRC of $1.5 \mathrm{~cm}^{2}$ to be fired onto ceramic surfaces, the authors searched for a best SQRC version that could store as much public and encrypted secret information as possible while enabling the easy extraction of the stored information. As a result of this search, we found that Ver. 3 can store the most character information (Fig. 3). Although the SQRC can store more information as the version number is increased, the cell size becomes smaller than that shown in Fig. 3. (Depending on the camera's resolution, extracting character information generally becomes difficult if the cell size is too small). In addition, unlike reading a printed SQRC (or observing reflected light from an SQRC), the camera observes light emission from the fired SQRC. Thus, the camera cannot capture each cell clearly if its resolution is low. Because the goal of this experiment is to confirm the feasibility of our idea, we adopted Ver. 3 for the SQRC to be fired onto the ceramics. However, in the future, we will study the correlation between the camera's resolution and the SQRC cell size.

\subsection{Public and secret information}

Table 2 shows an example of URLs linked to public and secret information. Each URL is also linked to the target individual(s), such as a consumer and verifier. Each directory has index.html as content (public or secret), but it only stores the directory information in the SQRC because each web client can receive the index.html (or content) from the web server by requesting only the directory information. For example, consumers and verifiers can access the public information of a product " $\mathrm{n} 1$ " by requesting www.abc123.co.jp/n1 on the web server because the content index.html (public information) is stored in the same directory. On the other hand, because the section chief 


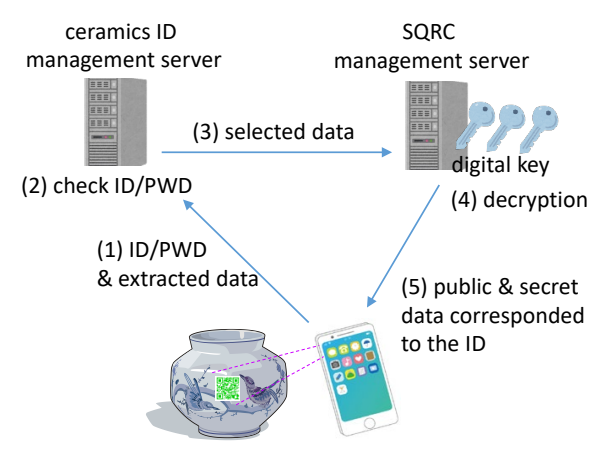

Fig. 4. Schematic of a network configuration and access control method.

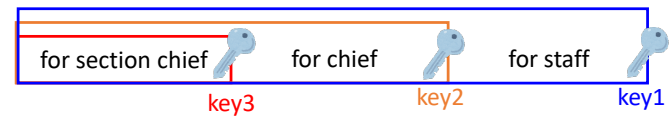

Fig. 5. Illustration showing the relationship between the encrypted secret information and corresponding digital key.

can access both public and secret information $(/ \sec 1, / \sec 2$, and $/ \sec 3$ ), he/she can read or refer to secret information (three verification methods to authenticate products).

\subsection{Access control of secret information}

Fig. 4 shows a network configuration and access control method for handling secret information. Entities in this framework are a smartphone, a ceramics identification (ID) management server, and an SQRC management server. The reader system for consumers does not have a function requesting an ID and a password, but the reader system for manufactures does. Because the ID and password varies for each verifier, the ceramics ID management server identifies a verifier by using the ID and password sent by the user. Next, the server extracts character information from the SQRC and sends both public information and ID-linked, encrypted, secret information to the SQRC management server. This server then decrypts the encrypted secret information by using a digital key linked to the ID and sends the decrypted secret information with the public information to a smartphone.

Fig. 5 shows the relationship between the encrypted secret information and the digital key. The secret information forms a nested structure and is encrypted by three digital keys. Secret information accessible by staff requires only one digital key, while secret information intended for the section chief needs three digital keys.

\subsection{SQRC printing and firing}

The SQRC with public and secret information is

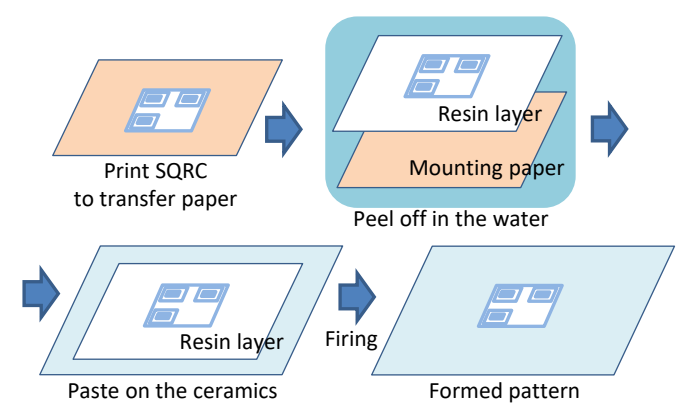

Fig. 6. Schematic of SQRC printing and firing.
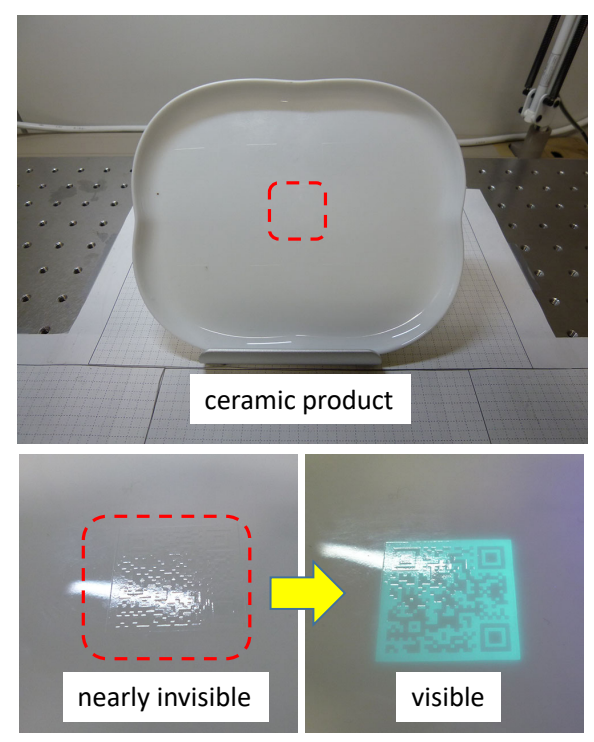

Fig. 7. A ceramic plate onto which an SQRC has been fired. The code is clearly visible under UV light.

printed on a transfer paper via screen-printing technology. Fig. 6 shows the process for printing the SQRC and firing it onto ceramic surfaces. First, a paste of paint is made from a squeeze oil, a glass frit, and a fluorescent pigment that emits visible light in response to UV light. The SQRC is printed on the transfer sheet with the paint. Next, the transfer paper is immersed in water so a transparent resin layer (a layer with the SQRC printed on its surface) can be peeled, pasted, and dried onto a product's surface. During this first firing, called glaze firing, a glassy layer is formed on the product's surface in a furnace through a glaze. After a second firing (decoration firing) in which a pigment is welded onto the ceramics, only the glass frit and fluorescent pigment remain. The welded glass frit fixes the pigment on the product surface and forms a glassy layer such that a nearly invisible SQRC is formed. Fig. 7 shows a product with fired and nearly invisible SQRC, and a clearly visible SQRC exposed to UV light. A clearly visible, pale-blue-colored SQRC can be seen on the product surface. Fired SQRC withstands rubbing with a brush while being 


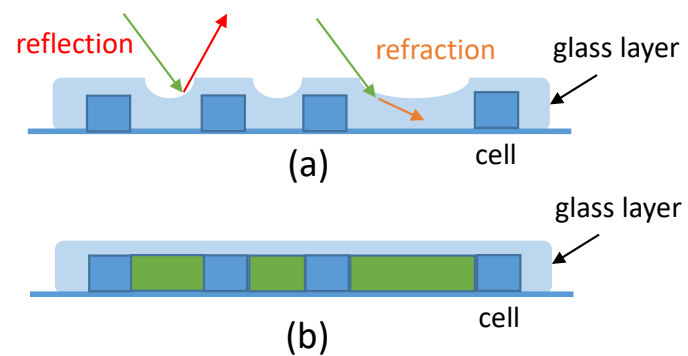

Fig. 8. (a) Reflection and refraction caused by the gaps between cells due to positive printing. (b) Positive and negative printing for filling the gaps.

washed because the SQRC is coated by a transparent glass layer.

The nearly invisible SQRC cannot be formed using ordinary screen-printing technology. As shown in Fig. 8(a), this printing method prints each cell (blue-colored block) on the resin layer. There is a gap between each cell, which would generate reflected and refracted lights. These lights emphasize the optical edge of the SQRC cells, enabling the fired SQRC to be distinguishable by the naked eye. To fill the gap, we made two plates (positive and negative, or reverse, SQRC pattern plates) and printed a gapless SQRC on the transfer paper (Fig. 8(b)). The positive plate prints blue-colored blocks (or cells), while the negative plate prints green-colored blocks. For the material to fill the gap (green-colored parts), we adopted strontium aluminate, instead of the fluorescent pigment, and used a mixture containing glass frit. We took this approach because this material represents the parent phase of the fluorescent pigment, and the material mixture could equal the refraction index and color tone of the mixture of the pigment and glass frit (blue-colored parts shown in Fig. 8(a)).

\section{Considerations}

\subsection{Handling a UV light source}

UV light is necessary for the proposed SQRC reading system to render the fired SQRC clearly visible on the ceramic surfaces. The code illuminates pale-blue light in the presence of UV light. The UV light, also called black light, is widely used not only in the industrial world but also in the education, cosmetics, and entertainment fields ${ }^{(3,}$ 4).

In general, commercially available UV light sources have a peak wavelength ranging from 350 to $380 \mathrm{~nm}$. These wavelengths are categorized as UVA and do not contain harmful wavelengths for the human body (below $320 \mathrm{~nm}$, categorized as UVB and UVC). Hence, commercial UV light sources are said to be safe compared to sunlight that consists of UV rays of 300 to $400 \mathrm{~nm}$ wavelengths ${ }^{(5)}$. However, manufacturers of ceramics with SQRCs should caution customers and verifiers against directly viewing the UV light sources with the naked eye to prevent any injuries. For example, a warning statement relating to the handling of the UV light source (e.g., "Do not look into the UV bulb") should be written on a printed product manual for customers and on an e-manual for verifiers to be used for product authentication.

\subsection{Identification of verifier}

Our SQRC reader app identifies each verifier by checking his/her ID and password as entered via their smartphone. Compared to a possession-based identification method, such as smartcards, this memory-based identification method (ID and password) does not require transporting any objects to be used for identification. However, it has some drawbacks, including the annoyance of inputting the ID and password, shoulder hacking (others peeping to steal the inputted ID and password), and forgetting the ID and password.

A biometrics-based identification method would maintain the advantage of the memory-based identification method while eliminating its drawbacks. Based on this viewpoint, the Fast Identity Online Alliance-a global industrial body for personal identification - promotes standardizing simple and secure identification methods (such as biometrics) to reduce dependency on the memory-based identification method ${ }^{(6)}$. Hence, in the future, the biometrics identification method could be a mainstream solution for personal identification. Currently, some smartphones on the market have a biometrics identification feature, and some of them have multimodal identification functions (identifying the user by checking multiple biometric parameters, such as face photos, iris scans, and fingerprints) ${ }^{(7)}$. In the future, we plan to link our SQRC reader app and smartphone-based biometric identification functions to further enhance security.

\section{Conclusions}

In this paper, we introduced a method for firing nearly invisible SQRCs to encode public and encrypted secret information onto ceramic surfaces. We set the product-related information as public information and the 
method for verifying the product's authenticity as secret information. Both consumers and verifiers can refer to the former, while only verifiers can access the latter. The secret information is divided into three categories, and each category contains different verification methods linked to the verifier's job position. Because the product and its public and secret information are linked through the SQRC, verifiers do not need to match each product to be verified and refer to the verification manual.

The gaps between SQRC cells, generated by ordinary screen printing, emphasize the cell edges. Thus, the code's invisibility was lowered due to light reflection and refraction. To cope with this issue, we made positive and negative SQRC plates and printed the code on the transfer paper. Using this technique, we fired nearly invisible SQRC onto the ceramic surfaces. The code could be made clearly visible with the illumination of pale-blue light from the fluorescent pigment in response to UV light irradiation (excitation light).

In the Considerations section, we discussed handling of the UV light source and identification method for each verifier. In the near future, we will link our SQRC reader app and biometrics-based identification functions installed in smartphones to enhance the system's security

\section{Acknowledgment}

This research was supported by the Strategic Core Technology Advancement Program FY28 (Supporting Industry Program), Chubu Bureau of Economy, Trade, and Industry, Japan. The authors received technical advice from Mr. Nakazawa and Mr. Hayashi of Denso Wave Incorporated. The terms "SQRC" and "QR code" are the above company's registered trademarks.

\section{References}

(1) Denso wave incorporated, "SQRC: A single code carries two types of data: public and private", " https://www.denso-wave.com/en/system/qr/product/sqr c.html

(2) Denso wave incorporated, "Error Correction Feature", https://www.qrcode.com/en/about/error correction.htm 1

(3) Educational Innovations, Inc. : "Ultraviolet Light Detecting Beads", http://cdn.teachersource.com/downloads/lesson_pdf/U V-AST.pdf
(4) Ushio America, Inc, : "UV-B, BL \& BLB", https:/www.ushio.com/files/specifications/blacklight-s pecialty-fluorescent.pdf

(5) Hiroaki Sasaki : "Shigaisen taisaku to ifuku (Japanese)", Society for Life Culture of Niigata, Vol. 17 , pp. 37-40, 2011

(6) Hidehito Gomi, and Wataru Oogami : "FIDO Authentication and Its Technology: Technical Specifications and Standardization Activities", IEICE Fundamentals Review, Vol. 12, No. 2, pp. 115-125, 2018

(7) Samsung Malaysia Electronics (SME) : "What is Intelligent Scan and how to use it?", https://www.samsung.com/my/support/mobile-devices/ what-is-intelligent-scan-and-how-to-use-it/, 2018 